\title{
Natural driving force
}

\author{
Ruiyu Zhu*1 \\ *Jiangnan University, Wuxi, P.R. China.
}

\begin{abstract}
We seem to be able to think about everything and imagine everything. However, we are just a higher form of natural evolution and cannot escape the shackles of nature. Our world is moving and changing. We inevitably feel that a force beyond our control drives everything and pushes everything forward. This artical discusses the natural driving force hidden in the objective world and the human body. There are five basic points: 1) Natural driving forces are everywhere and control everything; 2) the natural driving force from the inside out; 3 ) expectations about the outcome of human-related matters, determine the choice of cause; 4) the natural driving force for society moves from individual to society and therefore only a full realization of everyone's life will produce a better society; and, 5) the foundation of life's growth is to face reality and pain by acknowledging the driving forces..
\end{abstract}

One may reflect upon the world and perceive everything in isolation. One cannot help but feel that something indeterminate drives everything forward. In essence, the world is constantly changing and what drives this movement and change is the natural driving force. The search for essence also stems from the pursuit of the first driving force of nature. The process from seed to plant is actually a process of continuous generation of natural forces. As long as the environment is suitable, the seed will automatically grow into a plant without any human involvement. Imagine a scene in the forest. Spring is here and the seeds germinate and grow into plants. If we want to resist the process from seed germination to plant formation, there must be a confrontation with the forces of nature. If one looks deeper into the cause and effect relationship, the most insignificant link appears at the bottom. Its presentation is not driven by natural forces. It does not appear to be an inevitable connection, but rather a random presentation. The link may even be controlled manually. For example, a chemical molecule is necessary for the growth of seeds into plants. The expression of the chemical molecule is not affected by external causality. The entire process may be regulated through an adjustment to the molecule.

\section{Ubiquitous natural driving force}

The phenomena of human societies and the appearance of all matter also are driven by natural forces. Moreover, only natural forces can provide a constant source of power for this to occur in such a broad system that demands such a large amount of time and

\footnotetext{
${ }^{1}$ This is a working document which has not been peer reviewed yet. Please address any comment or remark to ry_zhu@hotmail.com or ry_zhu@sina.com.
} 
energy. Appearances have led societies to believe otherwise. How much time has been spent constantly fighting the forces of nature? What has been the result? Similar to when one weeds a wasteland, there may be a short-lived victory, but the problem will reappear. Much energy is spent in confrontation with natural forces.

Don't forget that God lets us exist, not accidentally, but naturally. The emergence of humankind is not because of a request, but because of the drive of natural forces. The desire for a better society to emerge from the problems societies confront, demands an identification of the causes that underlie complex phenomena. The root of the problem is best found through a focus of limited human energy on low-level phenomena as opposed to a confrontation with natural forces. This permits the full utilization of the energy provided by natural forces. Beauty becomes a passive presentation of nature, one that is truly stable and long-lasting.

Any problem that can be intuitively touched is also a natural presentation. Often one needs to check to understand if this representation is due to an unexpected event or to the continued natural forces that underlie it. If it is presented accidentally, there is no natural force responsible for its generation. Humans are capable of directly solving such problems. However, if there is a natural force behind the problem, its root causes must be identified. To do otherwise would result in only a temporary solution and more problems would arise; we cannot resist natural forces. The mining of the underlying natural forces requires a complicated thinking process. However, this process also is progressive; it moves humans forward.

Human brains tend to accumulate information. Additionally, memory increases the volume of information. Moreover, the conscious environment will naturally encourage the development of internal needs and external actions. All of these are driven by natural forces. The invisible power underlying a person's behavior is a built-in spiritual world. This spiritual world inevitably is influenced by experience. People can experience similar natural scenes, but their emotional states and the energy generated is different. The emotions related to the natural situation must be obtained through actual experience in order for relevant objective cognition to be achieved.

From this, we can see that natural forces dominate everything. All changes in our objective world are related to them. This effect is sometimes continuous or "regular." At times it is sporadic. When they are sporadic, humans are able to regulate it. Human energy is insignificant relative to natural power. The energy power that can be controlled is that of the body. Continuous energy is derived from that which is passed from generation to generation; the powerful energy that can be formed comes from the cooperation of many individuals. Human energy is limited and lacks the continuity of that found in the vastness of nature. The direction of our energy must be able to follow a natural course so that the results may be continuously presented under the influence of the natural driving force. Only in this way may the results obtained be preserved for a long time. Unfortunately humans rarely are aware of this. Rather, they often are swayed by emotions, confused by existing cognition, hostage to desires, and are in conflict with natural forces. The most powerful part of nature comes from this invisible natural driving force, not what we see in nature. Therefore, one should examine the problem from the perspective of movement, in order to truly grasp its essence. 


\section{The natural driving force for people is from the inside out}

The most fundamental source of material and power for human society are humans. However, when one looks at this world as an observer, it is easy to use the external background as a reference and as a criterion for judging, and forget the variability of the person. The ability to change and adapt to the environment represent people's most important faculties. To release the ability of a person is to enable the person to bloom from the inside out.Human behavior is controlled by the brain. That control is a natural process with mechanisms established by natural laws. An independent protective mechanism is formed during the onset of adulthood. It serves for the basic survival of human beings and the stability of the inner spiritual world. Imagine! If the brain were to receive external signals without self-protection, it would lose internal stability. A stable response demands coherence between an external signal and the internal environment of the brain. Only when the brain can produce a certain signal, can it respond to this signal. Moreover, this coherence permits the signal to remain unaffected by the inner resistance of the brain during the operation. The brain protects and conserves, creating a type of resistance to external signals. When the resistance has to be accepted, there is some internal discomfort, resulting in a further stimulation of the corresponding negative emotions. Therefore, one needs to pay attention to these natural reactions from the inside out. This permits us to address problems experienced with other people. Additionally, when the desire is to cultivate others' growth, there is a need to pay particular attention to the driving force from the inside out. Therefore, the problems that can be seen are not the real problems. Rather, the true challenge is to identify those that arise from within a person. When a person needs something from another, efforts should be focused on assuring that the expression of that need resonates with their natural presentation from the inside out. The attention to the direction of natural forces, yields pleasant and long-lasting outcomes.

Everyone's own development is an important foundation for social development. Social problems are best resolved with a commitment to the continued growth-from the inside out —of the people involved.

\section{The effect determines the cause}

Does the cause determine the effect or does the effect determine the cause? One often thinks that there is an inevitable causal connection between things. The unfolding of events over time encourages an observer to believe that cause determines effect. Cold weather causes plants to freeze. Bacterial infection causes food to spoil. These occur because there is a natural course of events without a corresponding adjustment process.

Human participation in events is different. The expectation of future effects encourages people to filter and intervene to achieve desired effects; this is a process of active regulation. The expectation with regard to effects determines the cause chosen. Before something comes to an end, humans can choose the cause to guide transformations in the anticipated direction. For example, when a person wants to have money, they might choose a job that permits them to make money. Or, when a person wants to eat more, they seek opportunities to obtain food. Most people's behaviors respond to the environment; the positive results obtained reinforce the behavior. During the Second World War, was the Nazi genocide the result of the disappearance of conscience or due to the environment at that time? People can 
choose and strengthen causes but cannot always control the specific process of causality. Rather, the process of moving from cause to result should be done by natural forces and rules. In other words, one needs to"actively choose and grow passively". The true life growth process is characterized by the adherence to a belief in good results. The active selection of causes allows natural energy to automatically flow, passively yielding beautiful fruits.

\section{Thinking about social problems from the perspective of individual existence}

One often relies on the environment as a reference point to observe people. Consequently, problems arise. An individual's life and existence should be taken as a starting point and a reference point for consideration; everything is based on existence. The existence of the individual determines the existence of the society. In order to achieve beautiful development for society, efforts must be based on the existence of individuals, so that the evolution process from individual to society becomes a spontaneous process, driven by natural forces. In other words, social growth should be a passive process. That is the only way that the society or a system may be more stable and exist longer. The energy and structure derived from nature can flow naturally. A willingness to give cannot be based on appearances because dedication may vary according to people's living conditions. It is cruel to demand the dedication of extremely poor people. It goes against natural forces. If a person is full of energy, then commitment will be a spontaneous process, a process of natural power. Their efforts will produce a sense of well-being. The major source of happiness comes from activities that follow the direction of natural forces. If it is not possible to dedicate or produce happiness at this time, it is a problem with values; it is necessary to first attend to a transformation of those values.

Natural forces control everything. Only when the direction of life or growth coincides with the direction of natural forces may humans create a better existence. Humans and human society can only regulate and control the existence or direction that is inconsistent with natural forces. Regulation is to regulate the disorder of existence.

\section{Facing reality and suffering is the foundation of growth}

All presentations are natural presentations. The most important thing for human growth is to allow one's own consciousness to continuously reflect the essence of reality and nature. Only in the face of nature can humans truly understand objective existence and be able to control the driving forces of nature. Hearts are often blocked by past painful experiences. Yet painful experiences do not reflect reality, but rather internal evaluation standards. Realities are the products of natural forces and have nothing to do with emotions. When pain occurs, one needs to confront and not avoid it. Pain is caused by the imperfection of our internal construction. It provides an opportunity for self-building and growth, not vice versa. One should learn to reshape their inner state, to shine a positive light on a wide variety of experience states. To do so is to ensure that a psychological barrier does not form and one may be better equipped to face reality and achieve growth. Additionally, the perfect standards established in our hearts, often hinder an acceptance and ability to face the true status quo. The recognition that perfection is an illusion proves difficult to achieve. Perfection is a kind of psychological virtual recognition, rather than a genuine reality. True reality can never set any standards, let alone form a perfect evaluation. Any existence is beautiful and is naturally presented. The beautiful standards set by 
humans are in fact only set by humans; they cannot be set by nature. There is a need to accept the imperfect self and not let imagination become a cognitive obstacle. Only then can one face challenges more realistically and better flourish. To face reality and suffering, and accept the imperfect self represent the foundations for growth for both individuals and society. It also is the foundation that permits a comprehension of the natural driving force.

\section{Natural driving force is the ultimate decisive force}

People are social first and then natural. Therefore, they need to withstand the double test of social rules and natural rules. Humans live in a social structure and will inevitably be bound by social rules. However, human beings - together with all material foundations - are created by nature and must endure the test of natural laws. The conflict between social rules and natural rules is an important source of human suffering. Because all material foundations of society come from nature, the social structure always has been shaped by the driving force of nature. Therefore, social rules eventually will be tested by natural laws. The conflict between social rules and the laws of natural movement is the root cause of the ebbs and flows observed in a variety of countries' histories. The social structure is subverted by the natural driving force transmitted by the laws of nature. Their appearance as war or social movements that overthrow existing regimes often result in disasters for humankind. 